\title{
Características clínicas da doença de Parkinson associadas à dor ao tratamento odontológico
}

\author{
Clinical characteristics of Parkinson's disease associated with dental \\ treatment-related pain \\ Etenildo Dantas CABRAL ${ }^{a *}$ (1) , Érica Alves da SILVA ${ }^{a}$ [D, Lícia Vasconcelos Carvalho da SILVA ${ }^{b}$ (D), \\ Carla Cabral dos Santos Accioly LINS ${ }^{c}$ (D), Maria das Graças Wanderley de Sales CORIOLANO` ${ }^{c}$ \\ aUFPE - Universidade Federal de Pernambuco, Faculdade de Odontologia, Recife, PE, Brasil \\ bUNITA - Centro Universitário Tabosa de Almeida, Caruaru, PE, Brasil \\ 'UFPE - Universidade Federal de Pernambuco, Programa de Pós-graduação em Gerontologia, Recife, PE, Brasil
}

Como citar: Cabral ED, Silva EA, Silva LVC, Lins CCSA, Coriolano MGWS. Características clínicas da doença de Parkinson associadas à dor ao tratamento odontológico. Rev Odontol UNESP. 2020;49:e20200070. https://doi.org/10.1590/1807-2577.07020

\begin{abstract}
Resumo
Introdução: A Doença de Parkinson (DP) resulta em condições clínicas desfavoráveis para a saúde bucal e não existe estudo publicado sobre dor ao tratamento odontológico em pacientes com DP. Objetivo: Pesquisar associação entre características clínicas da DP e a intensidade de dor em decorrência de tratamentos odontológicos. Material e método: Foi utilizada uma amostra consecutiva de 70 pacientes com DP, nos quais foram realizados 217 procedimentos odontológicos invasivos. Tempo de DP foi coletado do prontuário odontológico; estágio da DP foi definido por meio da escala de Hoehn e Yahr; défice cognitivo, por meio do Mini Exame do Estado Mental; depressão, por meio do Inventário de Depressão de Beck; e comprometimento motor, por meio da escala UPDRS/AVD. Os pacientes foram questionados se no último mês sentiram alguma sensação de dormência, formigamento, queimação ou dor bem como dificuldade para sentir cheiros ou gostos. A dor relacionada ao tratamento foi coletada por meio de uma escala numérica de dor com valores de 0 a 10 . Resultado: 0 nível de dor percebida pelos pacientes durante o tratamento odontológico não apresentou associação significativa com tempo de DP, estágio da DP, comprometimento motor nem ocorrência de depressão. Maior nível de dor foi estatisticamente mais frequente entre aqueles com défice cognitivo e aqueles que relataram a presença de maior número de sintomas sensitivos, sobretudo dormência e queimação. Conclusão: A dor ao tratamento odontológico em pacientes com DP está associada aos sintomas não motores desses pacientes, especificamente o défice cognitivo e a presença de sintomas sensitivos.
\end{abstract}

Descritores: Doença de Parkinson; dor; dor processual; percepção da dor; disfunção cognitiva.

\begin{abstract}
Introduction: Parkinson's disease (PD) results in unfavorable clinical conditions for oral health and there is no published study on pain during dental treatment in PD patients. Objective: To investigate association between clinical characteristics of PD and the intensity of pain due to dental treatments. Material and method: A consecutive sample of 70 patients with PD was used, in which 217 invasive dental procedures were performed. PD duration was collected from dental records; PD stage was defined using the Hoehn and Yahr scale; cognitive deficit, using the Mini Mental State Examination; depression, using the Beck Depression Inventory; and motor impairment, using the UPDRS-AVD scale. Patients were asked whether in the last month they felt any numbness, tingling, burning or pain sensation; as well as difficulty in smelling or tasting. Treatment-related pain was collected using a Numerical Pain Scale with values ranging from 0 to 10. Result: the level of pain perceived by patients during dental treatment was not significantly associated with PD duration, PD stage, motor impairment or occurrence of depression. The frequency of patients who reported a higher level of pain was statistically higher among those with cognitive impairment and those who reported the presence of a greater number of sensory symptoms, especially numbness and burning. Conclusion: Pain related to dental treatment in PD patients is associated with non-motor symptoms of these patients, specifically the cognitive impairment and the presence of sensory symptoms.
\end{abstract}

Descriptors: Parkinson's disease; pain; procedural pain; pain perception; cognitive dysfunction. 


\section{INTRODUÇÃO}

A Doença de Parkinson (DP) é a segunda desordem neurodegenerativa mais comum e a de maior crescimento no mundo ${ }^{1}$. Os sinais cardinais da DP estão relacionados à disfunção motora, mas os sintomas não motores são reconhecidos por desempenhar um papel importante ao afetar adversamente a qualidade de vida dos pacientes. Os sintomas não motores incluem distúrbios do sono, depressão, anormalidades cognitivas, distúrbios sensitivos e diferentes tipos de dor, dentre outros².

Apesar de a DP apresentar distintas formas de evolução, a presença e intensidade dos sintomas normalmente se acentuam com o passar do tempo e a progressão da doença ${ }^{3}$. Assim, período de tempo com a doença e estágio da doença são também características clínicas importantes na avaliação e estudo da DP.

Em decorrência das disfunções motoras e não motoras e dos efeitos colaterais das medicações para o tratamento da DP, os indivíduos com essa doença apresentam vários problemas orofaciais. A higiene bucal é severamente comprometida devido à falta de controle muscular, depressão e problemas cognitivos 4 .

O conjunto de problemas de saúde mencionados justifica a maior frequência de desgaste dental, doenças periodontais, cárie dentária e perda precoce de dentes nos indivíduos com DP apresentada na literatura ${ }^{4,5}$. Essa condição de saúde bucal desfavorável sugere uma maior necessidade de tratamentos odontológicos invasivos nessa população.

Quanto mais invasivos são os procedimentos odontológicos, mais os pacientes em geral referem dor ao tratamento ${ }^{6} .0$ medo de sentir essa dor pode retardar a ida de pessoas ao dentista7 ${ }^{7}$ o que piora suas condições de saúde bucal e, por sua vez, requer tratamentos mais invasivos, mais dolorosos. Esse ciclo vicioso pode ser agravado se a condição motora do paciente dificultar seu deslocamento para os consultórios odontológicos, como é o caso dos pacientes com DP. Desse modo, é de fundamental importância pesquisar a dor percebida pelos pacientes com DP durante os atendimentos odontológicos. Entretanto, estudos com esforços nesse sentido são ausentes na literatura.

Adicionalmente, identificar os sintomas que possam estar mais associados à percepção de dor durante o tratamento odontológico pode auxiliar o dentista a estimar o risco dessa dor e orientálo quanto ao seu modus operandi na busca de um tratamento indolor.

Portanto, considerando a alta prevalência da DP, a condição de saúde bucal desfavorável de indivíduos com essa doença, o impacto negativo que tratamentos dolorosos podem ter sobre essa condição de saúde e a carência da literatura científica sobre o tema, o objetivo do presente estudo foi verificar se existe associação entre algumas características clínicas de pacientes com DP e a intensidade de dor em decorrência de tratamentos odontológicos.

\section{MATERIAL E MÉTODO}

Este foi um estudo epidemiológico observacional, transversal e analítico. As características clínicas dos pacientes com DP analisadas, determinantes da dor ao tratamento odontológico, foram: o tempo de DP, o estágio da DP e alguns sintomas da DP. 0 termo "tempo de DP" refere-se ao lapso de tempo desde o diagnóstico da DP até o atendimento odontológico. Os sintomas da DP incluídos no estudo foram: comprometimento motor, défice cognitivo, depressão e sintomas sensitivos. Nesse último caso, foram considerados dormência, formigamento, queimação, dor, prejuízo olfativo e prejuízo gustativo.

\section{População e Amostra}

A pesquisa estudou os pacientes com DP assistidos pelo projeto de extensão "Pró-Parkinson: odontologia" durante o período de setembro de 2017 a junho de 2019. Os pacientes do projeto 
são residentes da região metropolitana de Recife - PE, sendo mais da metade das classes econômicas $\mathrm{C}$ e D e com até o primeiro grau incompleto.

Por se tratar de uma população pequena, foi utilizada uma amostragem consecutiva, ou seja, foram arrolados todos os pacientes que concordaram em participar da pesquisa e que atendiam aos critérios de seleção da amostra durante o período estipulado para a coleta dos dados. Isso resultou em uma amostra de 70 pacientes com DP e 217 procedimentos, alcançando um poder de $81 \%$ para detectar um tamanho de efeito de 0,5 por meio de um teste qui-quadrado com grau de liberdade 1 .

Foram incluídos os pacientes com diagnóstico clínico de DP idiopática que foram submetidos a procedimento odontológico do tipo invasivo, como exodontia e outras cirurgias, restauração, tartarectomia, raspagem periodontal e procedimentos endodônticos. Foram excluídos aqueles pacientes cuja condição de saúde indicasse muita dificuldade em responder à entrevista e/ou aqueles submetidos a procedimento odontológico do tipo não invasivo, como exame clínico, radiografias, orientação de higiene, profilaxia e moldagem.

Os pacientes foram atendidos na "fase on" ou "estado on", ou seja, foram aconselhados a tomar a medicação para DP 60 a 90 minutos antes do atendimento odontológico, de modo a oferecer tratamento mais confortável e seguro.

\section{Coleta de Dados}

As informações sobre o tempo de DP foram coletadas dos prontuários e fichas do serviço; o estágio da DP foi definido por meio da Escala de Estágios de Incapacidade de Hoehn, Yahr8; o défice cognitivo foi definido por meio do Mini Exame do Estado Mental - MEEM ${ }^{9}$; e a depressão foi identificada por meio do Inventário de Depressão de Beck para Atenção Primária - IBD-PC ${ }^{10}$.

0 comprometimento motor geral foi verificado por meio da parte II (atividades de vida diária) da Escala Unificada de Avaliação da Doença de Parkinson - UPDRS11, excluindo-se os itens 6 (salivação) e 17 (queixas sensitivas), o que se chamou de UPDRS/AVD-. A exclusão de tais itens deveu-se ao fato de não tratarem de questão motora, diferentemente de todos os demais.

Com o intuito de especificar o comprometimento motor na região orofacial, definiu-se o comprometimento da fala e da deglutição através dos itens específicos dessas funcionalidades na parte II do UPDRS. Em ambos os casos, pontuação 0 no item representou ausência de comprometimento e demais pontuações, presença de comprometimento.

Não se utilizou a parte III (exame motor) da UPDRS para determinação do comprometimento motor pelo fato de que essa requer conhecimentos teóricos e práticos que não são do âmbito da odontologia, sendo o UPDRS II mais factível para o cirurgião-dentista, o que favorece a utilização dos parâmetros aqui empregados na abordagem desses profissionais frente a seus pacientes com DP em ambiente clínico. Além do mais, o escore da parte II é menos sensível às alterações decorrentes do uso de medicação para a DP do que o da parte III, sendo bom indicador para avaliações na "fase on"12.

Para a verificação da existência de sintomas sensitivos, os pacientes foram questionados se: a) "No último mês, você tem sentido alguma sensação de dormência, formigamento, queimação, dor ou algo parecido no seu corpo que não seja por conta de outra doença que você sabe que tem (ex. artrite) nem por conta de uma pancada/acidente?"; b) "Você tem sentido dificuldade para sentir cheiros?"; c) "Você tem sentido dificuldade para sentir o gosto/sabor dos alimentos?".

A informação sobre a dor relacionada ao tratamento foi coletada logo após o atendimento, por meio de uma escala numérica de dor de 21 pontos, com a qual o paciente foi solicitado a indicar a intensidade da dor que sentiu, assinalando um $X$ ou verbalizando um número de 0 a 10 , com intervalos de 0,5. Quando e se o paciente foi submetido a outro procedimento invasivo, a informação sobre a dor ao tratamento foi verificada novamente, de maneira que se computou uma média de dor para cada paciente. 


\section{Análise dos Dados}

Os dados foram analisados através do programa estatístico SPSS versão 20 (IBM Corp., Armonk, Nova York, USA). A variável "número de sintomas sensitivos" foi computada, considerando-se a quantidade desses sintomas encontrados no mesmo paciente, e incluída para análise.

Por meio de uma análise exploratória, verificou-se que a intensidade de dor ao tratamento não apresentava distribuição normal (teste de Komolgorov-Smirnov) e que testes não paramétricos não elucidavam bem suas relações com outras variáveis. Assim sendo, essa variável foi categorizada, com base em sua média, em dois níveis: $<1,0$ e $\geq 1,0$.

O tempo de DP, o escore UPDRS/AVD- e o número de sintomas sensitivos também foram categorizadas em dois níveis, utilizando suas medianas como ponto de corte. Já o escore do MEEM foi categorizado utilizando o valor $\leq 24$ como ponto de corte por ser esse o valor mais recomendado como indicativo da presença de défice cognitivo. Simulações com pontos de corte um ponto acima ou abaixo dos que foram utilizados mostraram resultados similares.

Através de estatística analítica, foi verificada a existência ou não de associação significativa entre o nível de dor relacionada ao tratamento odontológico e as características clínicas da DP por meio do teste qui-quadrado. 0 nível de significância de $5 \%$ foi utilizado como referência em todos os testes estatísticos.

\section{Considerações Éticas}

Esta pesquisa foi realizada em acordo com princípios éticos contidos na Declaração de Helsinki e com a Resolução no 466/12 do Conselho Nacional de Saúde. Portanto, só foi realizada após aprovação do projeto de pesquisa pelo Comitê de Ética do Centro de Ciências da Saúde da Universidade Federal de Pernambuco (parecer no 1.330 .987 de novembro de 2015).

\section{RESULTADO}

A maioria dos pacientes da amostra era do sexo masculino $(64,3 \%)$ e foi submetida a procedimentos do tipo restauração $(44,2 \%)$ e raspagem periodontal $(32,3 \%)$. A idade média desses pacientes foi de $64( \pm 9)$ anos e a dor média relatada por eles foi de $0,9( \pm 1,1)$.

Os pacientes encontravam-se com tempo de DP de no mínimo 8 meses e no máximo 20 anos, com uma média de $7( \pm 4,3)$ anos. Quanto ao estágio da DP, 33,8\%, 56,9\% e 9,2\% estavam no estágio I, II e III, respectivamente. Não houve nenhum caso no estágio IV ou V.

A Tabela 1 mostra que o nível de intensidade de dor percebida pelos pacientes com DP durante o tratamento odontológico não apresentava associação com o tempo de DP $(p=0,76)$ nem com o estágio da DP $(p=0,90)$ em que esses pacientes se encontravam. Também não estava associado ao comprometimento motor dos referidos pacientes, seja avaliando especificamente o comprometimento de fala $(p=0,25)$, de deglutição $(p=0,63)$ ou mesmo por meio do escore geral do UPDRS/AVD- $(\mathrm{p}=0,95)$, ou seja, a dor percebida pelos pacientes com DP não foi estatisticamente diferente segundo o tempo de doença, o estágio da doença nem o comprometimento motor.

Ainda na Tabela 1 pode-se verificar que a frequência de pacientes com DP que referiram maior nível de intensidade de dor foi mais alta entre aqueles com défice cognitivo $(54,4 \%)$ e aqueles com depressão $(53,3 \%)$ comparada com aqueles sem défice cognitivo $(27,7 \%)$ e sem depressão $(31,5 \%)$. Entretanto, essas diferenças só foram estatisticamente significativas no caso do défice cognitivo $(p=0,03)$. 
Tabela 1. Frequência de diferentes níveis de dor relativa ao tratamento odontológico segundo tempo e estágio da doença, comprometimento motor, cognição e depressão de pacientes com Doença de Parkinson

\begin{tabular}{|c|c|c|c|c|c|c|}
\hline \multirow{3}{*}{ Característica } & & \multicolumn{4}{|c|}{ Nível de dor } & \multirow{3}{*}{$\begin{array}{c}\text { Valor de } p \\
\qquad\left(X^{2}\right)\end{array}$} \\
\hline & & \multicolumn{2}{|c|}{$<1,0$} & \multicolumn{2}{|c|}{$\geq 1,0$} & \\
\hline & & $\mathbf{N}$ & $\%$ & $\mathbf{N}$ & $\%$ & \\
\hline \multirow{2}{*}{ Tempo de DP } & $<6$ anos & 17 & 60,7 & 11 & 39,3 & \multirow{2}{*}{0,76} \\
\hline & $\geq 6$ anos & 27 & 64,3 & 15 & 35,7 & \\
\hline \multirow{2}{*}{ Estágio da DP* } & I & 14 & 63,6 & 8 & 36,4 & \multirow{2}{*}{0,90} \\
\hline & II ou III & 28 & 65,1 & 15 & 34,9 & \\
\hline \multirow{2}{*}{ Comprometimento da fala } & Não & 16 & 72,7 & 6 & 23,7 & \multirow{2}{*}{0,25} \\
\hline & Sim & 28 & 58,3 & 20 & 41,7 & \\
\hline \multirow{2}{*}{$\begin{array}{c}\text { Comprometimento da } \\
\text { deglutição }\end{array}$} & Não & 28 & 60,9 & 18 & 39,1 & \multirow{2}{*}{0,63} \\
\hline & Sim & 16 & 66,7 & 8 & 33,3 & \\
\hline \multirow{2}{*}{ Escore UPDRS/AVD-** } & $<11$ & 20 & 62,5 & 12 & 37,5 & \multirow{2}{*}{0,95} \\
\hline & $\geq 11$ & 24 & 63,2 & 14 & 36,8 & \\
\hline \multirow{2}{*}{ Escore MEEM $^{* * *}$} & $\leq 24$ & 10 & 45,5 & 12 & 54,4 & \multirow{2}{*}{0,03} \\
\hline & $>24$ & 34 & 72,3 & 13 & 27,7 & \\
\hline \multirow{2}{*}{ Depressão } & Não & 37 & 68,5 & 17 & 31,5 & \multirow{2}{*}{0,12} \\
\hline & Sim & 7 & 46,7 & 8 & 53,3 & \\
\hline
\end{tabular}

* Segundo a escala de Hoehn e Yahr. Informação não obtida para alguns pacientes. ** Parte II (AVD) da Escala Unificada de Avaliação da Doença de Parkinson (UPDRS), excluindo-se os itens 6 e 17. Maior o escore, maior o comprometimento motor. *** Mini Exame do Estado Mental. Menor o escore, maior o défice cognitivo.

Conforme visto na Tabela 2, maior nível de intensidade de dor ao tratamento odontológico foi também mais frequente entre aqueles pacientes com DP que relataram a presença de sintomas sensitivos, sobretudo dormência (53,6\%), queimação (64,3\%), dor (45,2\%) e prejuízo gustativo (50\%), que entre aqueles sem esses sintomas. Nesses casos, as diferenças chegaram a ser estatisticamente significativas para os sintomas de dormência e queimação $(\mathrm{p}=0,02$ para ambos). Considerando o número de sintomas sensitivos existentes nos pacientes com DP, aqueles com maior número desses sintomas relataram maior nível de dor com mais frequência $(51,7 \%)$ que aqueles com menor número de sintomas sensitivos (26,8\%), sendo essa diferença estatisticamente significativa $(p=0,03)$.

Tabela 2. Frequência de diferentes níveis de dor relativa ao tratamento odontológico segundo sintomas sensitivos de pacientes com Doença de Parkinson

\begin{tabular}{|c|c|c|c|c|c|c|}
\hline \multirow{3}{*}{ Sintomas } & & \multicolumn{4}{|c|}{ Nível de dor } & \multirow{3}{*}{$\begin{array}{c}\text { Valor de p } \\
\left(X^{2}\right)\end{array}$} \\
\hline & & \multicolumn{2}{|c|}{$<1,0$} & \multicolumn{2}{|c|}{$\geq 1,0$} & \\
\hline & & $\mathbf{N}$ & $\%$ & $\mathbf{N}$ & $\%$ & \\
\hline \multirow{2}{*}{ Dormência } & Não & 31 & 73,8 & 11 & 26,2 & \multirow{2}{*}{0,02} \\
\hline & $\operatorname{Sim}$ & 13 & 46,4 & 15 & 53,6 & \\
\hline \multirow{2}{*}{ Formigamento } & Não & 27 & 65,9 & 14 & 34,1 & \multirow{2}{*}{0,53} \\
\hline & $\operatorname{Sim}$ & 17 & 58,6 & 12 & 41,4 & \\
\hline \multirow{2}{*}{ Queimação } & Não & 39 & 69,6 & 17 & 30,4 & \multirow{2}{*}{0,02} \\
\hline & Sim & 5 & 35,7 & 9 & 64,3 & \\
\hline \multirow{2}{*}{ Dor } & Não & 21 & 75,0 & 7 & 25,0 & \multirow{2}{*}{0,08} \\
\hline & Sim & 23 & 54,8 & 19 & 45,2 & \\
\hline \multirow{2}{*}{ Prejuízo olfativo } & Não & 21 & 65,6 & 11 & 34,4 & \multirow{2}{*}{0,66} \\
\hline & $\operatorname{Sim}$ & 23 & 60,5 & 15 & 39,5 & \\
\hline \multirow{2}{*}{ Prejuízo gustativo } & Não & 32 & 69,6 & 14 & 30,4 & \multirow{2}{*}{0,10} \\
\hline & $\operatorname{Sim}$ & 12 & 50,0 & 12 & 50,0 & \\
\hline \multirow{2}{*}{ Número de sintomas sensitivos } & $\leq 2$ & 30 & 73,2 & 11 & 26,8 & \multirow{2}{*}{0,03} \\
\hline & $>2$ & 14 & 48,3 & 15 & 51,7 & \\
\hline
\end{tabular}




\section{DISCUSSÃO}

Inicialmente é necessário esclarecer que, haja vista a ausência de pesquisas com o mesmo tipo de população e objetivo do estudo atual, não existem dados disponíveis na literatura para uma comparação direta com os resultados aqui encontrados. Sendo assim, as discussões foram feitas com base em estudos afins. Por exemplo, estudos experimentais sobre limiar, tolerância e sensibilidade à dor em indivíduos com ou sem DP e estudos clínicos de dor aguda em indivíduos sem DP. Enfim, os estudos disponíveis que de alguma forma relacionavam dor com as variáveis aqui analisadas.

Os resultados encontrados no presente estudo não mostraram associação entre o tempo de DP e a dor relatada pelos pacientes diante do tratamento odontológico, cabendo algumas considerações sobre esse aspecto. Primeiramente, vale dizer que tempo de DP não tem relação linear direta com a gravidade da DP. Existem pacientes com uma progressão rápida da doença e outros com progressão lenta ${ }^{13}$. Segundo, porém não menos importante, é que estudos afins mostram resultados na mesma direção dos aqui encontrados. Por exemplo, uma meta-análise avaliando estudos que comparavam grupo com DP e grupo controle em suas respostas a estímulos dolorosos experimentais indicou não haver relação entre limiar/intensidade de dor e o tempo de DP14.

A ausência de associação entre a dor relatada pelo paciente e o estágio da DP identificada neste estudo é conflitante com um estudo que observou limiar de dor e de tolerância à dor de indivíduos com DP correlacionarem-se significativamente com o estágio da doença ${ }^{15}$. Alguns aspectos talvez expliquem, em parte, esse dado conflitante. Um deles seria a baixíssima frequência de casos no estágio III e nenhum nos estágios IV ou V no estudo atual, o que não permitiu a comparação entre estágios iniciais e estágios mais avançados (I vs. IV, por exemplo). Outro aspecto a ser considerado é a diferença na metodologia do citado estudo ${ }^{15}$ frente ao atual. 0 primeiro não avaliou dores em regiões faciais e, sobretudo, os indivíduos foram todos avaliados em "estado off". Como tem sido demonstrado na literatura, a diminuição do limiar de dor presente na DP pode ser atenuada pela administração de dopamina ("estado on") 14,16.

O comprometimento motor do paciente, seja avaliado por meio do escore geral do UPDRS/AVD- ou de seus componentes específicos de fala e deglutição, também não apresentou associação com a dor relativa ao tratamento odontológico. Esses resultados são coerentes com estudos que mostraram a não relação do comprometimento motor com o limiar de dor a estímulos dolorosos ${ }^{14}$. Tem sido sugerido que os sintomas motores e não motores não compartilham necessariamente os mesmos mecanismos, não havendo correlação direta entre alterações sensoriais/dolorosas e motoras ${ }^{16}$.

Quanto à maior intensidade de dor tendo sido relatada entre aqueles pacientes com maior défice cognitivo, é necessário lembrar, inicialmente, que a dor não é somente a transmissão do estímulo de uma parte do corpo para a medula espinhal e para o cérebro, mas que também há modulação através dos aspectos cognitivos. Explicitando melhor essa relação entre dor e défice cognitivo, uma extensa revisão de literatura que pesquisou diversas desordens neurológicas mostrou que, independentemente da etiologia do comprometimento cognitivo, pacientes nessa condição geralmente sentem estímulos nocivos com hipersensibilidade em comparação com indivíduos sem comprometimento cognitivo ${ }^{17}$. No que concerne especificamente à DP, sabe-se que a função anormal dos núcleos basais nos indivíduos com essa doença não interfere apenas na propagação dos sinais nociceptivos, mas também nos processos cognitivos e afetivos da dor ${ }^{18}$. Reforçando essa tese e corroborando os achados da presente pesquisa, um estudo identificou que a anormalidade no processamento da dor, determinada por potenciais evocados somatossensitivos, correlacionou-se significativamente com o défice cognitivo em pacientes com DP ${ }^{19}$.

A relação entre dor crônica e depressão está bem estabelecida. Mas estudos que relacionem depressão com dor aguda não são numerosos. Concernente à dor induzida experimentalmente, existem dados conflitantes com estudos que encontraram pacientes com depressão apresentando 
menor limiar de dor enquanto outros não ${ }^{20}$. Porém, mesmo quando não se identificou menor limiar de dor, os pacientes com depressão apresentaram maior sensibilidade a dor 21. Estudos clínicos também mostraram forte correlação entre intensidade de dor aguda e depressão, incluindo dor relacionada a tratamentos odontológicos ${ }^{22}$. No âmbito da DP, estudos encontraram que a depressão tem associação significativa e relação inversa com o limiar e a tolerância a dor ${ }^{15}$. Portanto, apesar do estudo atual não ter alcançado significância estatística no nível de 5\% para a associação entre a dor ao tratamento odontológico e a depressão, parece coerente manter atenção a essa variável.

Os sintomas de dor na DP podem ser classificados em cinco categorias: musculoesquelética, radicular/neuropática, relacionada à distonia, desconforto/dor acatísica e dor central ou primária. A dor primária apresenta características neuropáticas, mas não está relacionada a uma lesão no sistema periférico e, sim, parece estar associada a um defeito na modulação central da dor $^{23}$. São descritas por 40 a 50\% dos pacientes com DP e englobam dormência, formigamento, queimação, frio, calor e dor. Importante característica da dor primária é a presença de alodínia, sensação dolorosa provocada por estímulo não doloroso, e hiperalgesia, sensação exagerada de dor provocada por estímulo doloroso de baixa intensidade 24 .

Um estudo observou sinais de hiperalgesia em pacientes com DP com dor primária. Devido ao fato de algumas anormalidades terem sido parcialmente modificadas após a administração de levodopa, os autores sugeriram que doentes de Parkinson com dor primária talvez sofram de uma disfunção ao nível de estruturas neurais dependentes de dopamina que regulam funções de modulação inibitória de impulsos dolorosos ${ }^{25}$. Os resultados encontrados no presente estudo sugerem também uma relação entre a tolerância à dor e os sintomas sensitivos da DP, principalmente com sintomas de dor primária, como dormência e queimação.

Como limitação do presente estudo, pode-se considerar o tamanho pequeno da amostra para os padrões de estudos epidemiológicos de associação estatística. Essa limitação se justifica pela dificuldade inerente de indivíduos com DP de se dirigirem aos serviços odontológicos. Em estágios mais avançados da DP, esses indivíduos podem depender totalmente de terceiros para a sua locomoção, o que justifica também outra limitação do estudo, que foi a carência de pacientes em estágios mais avançados.

Em que pese as limitações, há de se considerar a relevância científica e importância clínica deste estudo. Esse foi o primeiro estudo que avaliou a relação entre dor ao tratamento odontológico e os sintomas da DP. Os dados e reflexões aqui apresentados contribuem para que os cirurgiões-dentistas zelem por uma assistência mais indolor aos pacientes com DP. Através disso, o paciente se sentirá mais confortável para buscar a assistência odontológica, o que favorece a sua saúde bucal.

\section{CONCLUSÃO}

A dor relacionada ao tratamento odontológico em pacientes com DP está associada a características clínicas dessa doença, em especial, à presença de sintomas não motores. Desses sintomas, o défice cognitivo, a dormência e a queimação parecem ser os mais significativos.

\section{REFERÊNCIAS}

1. Dorsey ER, Sherer T, Okun MS, Bloem BR. The emerging evidence of the Parkinson Pandemic. J Parkinsons Dis. 2018;8(s1):S3-8. http://dx.doi.org/10.3233/JPD-181474. PMid:30584159.

2. Lee A, Gilbert RM. Epidemiology of Parkinson Disease. Neurol Clin. 2016 Nov;34(4):955-65. http://dx.doi.org/10.1016/j.ncl.2016.06.012. PMid:27720003. 
3. Vu TC, Nutt JG, Holford NHG. Progression of motor and nonmotor features of Parkinson's disease and their response to treatment. Br J Clin Pharmacol. 2012 Aug;74(2):267-83. http://dx.doi.org/10.1111/j.1365-2125.2012.04192.x. PMid:22283961.

4. Zlotnik Y, Balash Y, Korczyn AD, Giladi N, Gurevich T. Disorders of the oral cavity in Parkinson's Disease and Parkinsonian Syndromes. Parkinsons Dis. 2015;2015:379482. http://dx.doi.org/10.1155/2015/379482. PMid:25685594.

5. Cicciù M, Risitano G, Lo Giudice G, Bramanti E. Periodontal health and caries prevalence evaluation in patients affected by Parkinson's Disease. Parkinsons Dis. 2012;2012:541908. http://dx.doi.org/10.1155/2012/541908. PMid:23320249.

6. Cabral ED, Alves GG, Souza GC. Dor durante o atendimento odontológico em unidades de saúde da família do município de Caruaru-PE. Rev Dor. 2013 Jun;14(2):100-5. http://dx.doi.org/10.1590/S1806-00132013000200005.

7. Kanegane K, Penha SS, Borsatti MA, Rocha RG. Dental anxiety in an emergency dental service. Rev Saude Publica. 2003 Dec;37(6):786-92. http://dx.doi.org/10.1590/S0034-89102003000600015. PMid:14666310.

8. Hoehn MM, Yahr MD. Parkinsonism: onset, progression, and mortality. Neurology. 1967 May;17(5):427-42. http://dx.doi.org/10.1212/WNL.17.5.427. PMid:6067254.

9. Folstein MF, Folstein SE, Mchugh PR. "Mini-mental state": a practical method for grading the cognitive state of patients for the clinician. J Psychiatr Res. 1975;12(3):189-98. http://dx.doi.org/10.1016/0022-3956(75)90026-6. PMid:1202204.

10. Steer RA, Cavalieri TA, Leonard DM, Beck AT. Use of the Beck depression inventory for primary care to screen for major depression disorders. Gen Hosp Psychiatry. 1999 Mar-Apr;21(2):106-11. http://dx.doi.org/10.1016/S0163-8343(98)00070-X. PMid:10228890.

11. Goetz CG, Tilley BC, Shaftman SR, Stebbins GT, Fahn S, Martinez-Martin P, et al. Movement Disorder Society-sponsored revision of the Unified Parkinson's Disease Rating Scale (MDS-UPDRS): scale presentation and clinimetric testing results. Mov Disord. 2008 Nov;23(15):2129-70. http://dx.doi.org/10.1002/mds.22340. PMid:19025984.

12. Harrison MB, Wylie SA, Frysinger RC, Patrie JT, Huss DS, Currie LJ, et al. UPDRS activity of daily living score as a marker of Parkinson's disease progression. Mov Disord. 2009 Jan;24(2):224-30. http://dx.doi.org/10.1002/mds.22335. PMid:18951537.

13. Roede JR, Uppal K, Park Y, Lee K, Tran V, Walker D, et al. Serum metabolomics of slow vs. rapid motor progression Parkinson's disease: a pilot study. PLoS One. 2013 Oct;8(10):e77629. http://dx.doi.org/10.1371/journal.pone.0077629. PMid:24167579.

14. Thompson T, Gallop K, Correll CU, Carvalho AF, Veronese N, Wright E, et al. Pain perception in Parkinson's disease: a systematic review and meta-analysis of experimental studies. Ageing Res Rev. 2017 May;35:74-86. http://dx.doi.org/10.1016/j.arr.2017.01.005. PMid:28179128.

15. Zambito Marsala S, Tinazzi M, Vitaliani R, Recchia S, Fabris F, Marchini C, et al. Spontaneous pain, pain threshold, and pain tolerance in Parkinson's disease. J Neurol. 2011 Apr;258(4):627-33. http://dx.doi.org/10.1007/s00415-010-5812-0. PMid:21082324.

16. Cury RG, Galhardoni R, Fonoff ET, Perez Lloret S, Dos Santos Ghilardi MG, Barbosa ER, et al. Sensory abnormalities and pain in Parkinson disease and its modulation by treatment of motor symptoms. Eur J Pain. 2016 Feb;20(2):151-65. http://dx.doi.org/10.1002/ejp.745. PMid:26147660.

17. Defrin R, Amanzio M, de Tommaso M, Dimova V, Filipovic S, Finn DP, et al. Experimental pain processing in individuals with cognitive impairment: current state of the science. Pain. 2015 Aug;156(8):1396-408. http://dx.doi.org/10.1097/j.pain.0000000000000195. PMid:26181216.

18. Truini A, Frontoni M, Cruccu G. Parkinson's disease related pain: a review of recent findings. J Neurol. 2013 Jan;260(1):330-4. http://dx.doi.org/10.1007/s00415-012-6754-5. PMid:23180185. 
19. Okada A, Nakamura T, Suzuki J, Suzuki M, Hirayama M, Katsuno M, et al. Impaired pain processing correlates with cognitive impairment in Parkinson's Disease. Intern Med. 2016 Nov;55(21):3113-8. http://dx.doi.org/10.2169/internalmedicine.55.7067. PMid:27803403.

20. Michaelides A, Zis P. Depression, anxiety and acute pain: links and management challenges. Postgrad Med. 2019 Sep;131(7):438-44. http://dx.doi.org/10.1080/00325481.2019.1663705. PMid:31482756.

21. Hermesdorf M, Berger K, Baune BT, Wellmann J, Ruscheweyh R, Wersching H. Pain sensitivity in patients with major depression: differential effect of pain sensitivity measures, somatic cofactors, and disease characteristics. J Pain. 2016 May;17(5):606-16. http://dx.doi.org/10.1016/j.jpain.2016.01.474. PMid:26867484.

22. Barman D, Mishra S, Mishra J, Mahapatra P, Manjareeka M. Association between depression and acute pain in adults attending a tertiary care hospital in Bhubaneswar. J Clin Diagn Res. 2015 Jul;9(7):CC0811. http://dx.doi.org/10.7860/JCDR/2015/12008.6179. PMid:26393121.

23. Blood MRY, Ferro MM, Munhoz RP, Teive HAG, Camargo CHF. Classification and characteristics of pain associated with Parkinson's disease. Parkinsons Dis. 2016;2016:6067132. http://dx.doi.org/10.1155/2016/6067132. PMid:27800210.

24. Garcia JBS, Barbosa JO No, Amâncio EJ, Andrade ETF. Central neuropathic pain. Rev Dor. 2016;17(Suppl 1):S67-71. http://dx.doi.org/10.5935/1806-0013.20160052.

25. Schestatsky P, Kumru H, Valls-Solé J, Valldeoriola F, Marti MJ, Tolosa E, et al. Neurophysiologic study of central pain in patients with Parkinson disease. Neurology. 2007 Dec;69(23):2162-9. http://dx.doi.org/10.1212/01.wnl.0000295669.12443.d3. PMid:18056580.

\section{CONFLITOS DE INTERESSE}

Os autores declaram não haver conflitos de interesse.

\section{*AUTOR PARA CORRESPONDÊNCIA}

Etenildo Dantas Cabral, UFPE - Universidade Federal de Pernambuco, Avenida Prof. Moraes Rego, 1235, Cidade Universitária, 50670-901 Recife - PE, Brasil, e-mail: etenildo.cabral@ufpe.br

Recebido: Outubro 19, 2020

Aprovado: Outubro 28, 2020 